\title{
Chaos-assisted emission from asymmetric resonant cavity microlasers
}

\author{
Susumu Shinohara, ${ }^{1}$ Takahisa Harayama, ${ }^{2}$ Takehiro Fukushima ${ }^{3}$ \\ Martina Hentschel, ${ }^{1}$ Satoshi Sunada, ${ }^{2}$ and Evgenii E. Narimanov ${ }^{4}$ \\ ${ }^{1}$ Max-Planck-Institut für Physik Komplexer Systeme, Nöthnitzer Straße 38, D-01187 Dresden, Germany \\ ${ }^{2}$ NTT Communication Science Laboratories, NTT Corporation, 2-4 Hikaridai, Seika-cho, Soraku-gun, Kyoto 619-0237, Japan \\ ${ }^{3}$ Department of Communication Engineering, Okayama Prefectural University, 111 Kuboki, Soja, Okayama 719-1197, Japan \\ ${ }^{4}$ Birck Nanotechnology Center, Department of Electrical and Computer Engineering, \\ Purdue University, 1205 West State Street West Lafayette, Indiana 47907-2057, USA
}

\begin{abstract}
We study emission from quasi-one-dimensional modes of an asymmetric resonant cavity that are associated with a stable periodic ray orbit confined inside the cavity by total internal reflection. It is numerically demonstrated that such modes exhibit directional emission, which is explained by chaos-assisted emission induced by dynamical tunneling. Fabricating semiconductor microlasers with the asymmetric resonant cavity, we experimentally demonstrate the selective excitation of the quasi-one-dimensional modes by employing the device structure to preferentially inject currents to these modes and observe directional emission in good accordance with the theoretical prediction based on chaos-assisted emission.
\end{abstract}

PACS numbers: 42.55.Sa, 05.45.Mt, 42.60.Da, 42.55.Px

\section{INTRODUCTION}

Besides growing interest from the fundamental physics of light and practical applications in photonics [1] 4 ], twodimensional optical microcavities have attracted much attention from the field of quantum/wave chaos, since they can be regarded as physical realization of dynamical billiards, a well-studied model in the field (for reviews see for example Refs. [5-7]). Concepts of the quantum/wave chaos theory have been successfully applied to two-dimensional microcavities to explain how ray chaos influences the characteristics of resonant modes such as emission directionality and lifetimes.

One of such applied concepts is dynamical tunneling [8], which refers to tunneling between classical invariant components disconnected from each other under classical dynamics (see Ref. [9] for a review). In generic Hamiltonian systems, i.e., those with mixed phase space, where regular and chaotic dynamics coexist, dynamical tunneling manifests itself in the fundamental processes such as regular-to-chaotic and chaotic-to-regular tunneling 10 13]. One of the dramatic effects of dynamical tunneling is observed in the enhancement of level splitting of doublets associated with symmetry-related regular components separated by a global chaotic sea. The phenomenon is explained by the mechanism known as chaos-assisted tunneling 14 19]; the regular components are coupled not only by direct regular-to-regular tunneling but also by a multi-step process involving regular-to-chaotic and chaotic-to-regular tunneling, and this process leads to a significant enhancement of the coupling. The dynamical tunneling and chaos-assisted tunneling have been experimentally observed in microwave billiards [20, 21] and cold atom systems [22, 23].

In the context of optical microcavities, the effect of dynamical tunneling was already pointed out in the pioneering theoretical study of asymmetric resonant cavities [24], where the significant shortening of resonance life- times is attributed to dynamical tunneling. This effect was quantitatively studied by effective Hamiltonian models 25 27], where a formula is derived describing how resonance lifetimes depend on the factors such as the area of regular components and wavenumber. Strong influence of dynamical tunneling on the emission pattern of a ray-chaotic cavity was also pointed out in Refs. [26, 27]. Resonance lifetimes were connected with a direct regularto-chaotic tunneling rate in Ref. [28]. The enhancement and fluctuation of frequency splitting of regular mode doublets is explained by the chaos-assisted tunneling in Ref. 29].

Recently, the manifestation of dynamical tunneling was experimentally observed in the emission patterns of semiconductor microlasers [30, 31] and the pumpcoupling efficiency of dye-doped liquid jet microlasers [32]. In the former study, an asymmetric resonant cavity was designed to have quasi-one-dimensional modes strongly localized along a stable periodic ray orbit that is confined by total internal reflection (TIR). In the experiment, such modes were excited by selective pumping, and highly directional emission was observed. The purpose of this paper is to present a comprehensive study on this microlaser. The emission directionality of this microlaser was interpreted by the following two steps: (i) dynamical tunneling from the TIR-confined ray orbit to a neighboring chaotic ray orbit, and (ii) ray-dynamical propagation following the chaotic dynamics leading to refractive emission. In this paper, we shall call this process chaos-assisted emission in order to emphasize that the process is regular-to-chaotic-to-continuum.

This paper is organized as follows: In Sec. П we introduce the asymmetric resonant cavity and explain its ray dynamics, where particular attention is paid to the rectangle-shaped stable ray orbit confined by TIR. Section III presents the numerical study of the resonant modes associated with this orbit (rectangle-orbit modes, in short), showing that these quasi-one-dimensional 
modes exhibit chaos-assisted directional emission. Section IV] explains the device structure of fabricated semiconductor microlasers, especially focusing on the structure to achieve selective excitation of the rectangle-orbit modes. Section $\nabla$ provides measured spectral data that evidence the selective excitation. Section VI presents measured emission patterns which are attributed to the chaos-assisted emission. Finally, our conclusion is given in Sect. VII

\section{ASYMMETRIC RESONANT CAVITY}

In this paper, we consider the asymmetric resonant cavity whose shape is defined in the polar coordinates $(r, \phi)$ as

$$
r=R(\phi)=r_{0}(1+a \cos 2 \phi+b \cos 4 \phi+c \cos 6 \phi),
$$

where $r_{0}$ is the size parameter and $a, b$, and $c$ are deformation parameters fixed as $a=0.1, b=0.01$, and $c=0.012$. The geometry of the cavity is depicted in Fig. 1 (a).

An important feature of this cavity is the existence of the 4-bounce stable ray orbit drawing the "rectangle" as shown in Fig. 1 (b), which is confined by TIR when the refractive index $n>\sqrt{2}$. The peculiarity of the rectangular orbit in relation with global ray dynamics is revealed by plotting the Poincaré surface of section (PSOS) of the ray dynamics. The PSOS describes the successive bounces of a ray trajectory usually using the Birkhoff coordinates $(s, \sin \theta)$, where $s$ represents the arclength along the cavity and $\theta$ the incident angle as illustrated in Fig. 1 (a). The PSOS is convenient for describing the openness of the dielectric cavity, because the condition for TIR is given by $|\sin \theta|>1 / n$. Throughout this paper, we assume $n=3.3$. This value corresponds to the effective refractive index calculated for the structure of actually fabricated devices to be explained in Sect. IV.

Because the PSOS is symmetric under $\sin \theta \mapsto-\sin \theta$, we show only the upper half of the PSOS in Fig. 1 (c), where one finds that ray dynamics is mostly chaotic, and the four islands of stability corresponding to the rectangular orbit are the only dominant islands located in the TIR-region, i.e., $\sin \theta>1 / 3.3$. In this paper, we focus on the resonant modes that are strongly localized on the TIR-confined dominant islands. The ray-dynamical property that these islands are isolated and only dominant islands in the global chaotic sea will later enable us to interpret the emission patterns of those resonant modes by a simple scenario based on chaos-assisted emission.

\section{RECTANGLE-ORBIT MODES}

Resonant modes of the cavity are given as the solutions of the Helmholtz equation derived from Maxwell's equations [5, 6, 24]. They are quasibound states with finite lifetimes characterized by complex wavenumbers $k$.
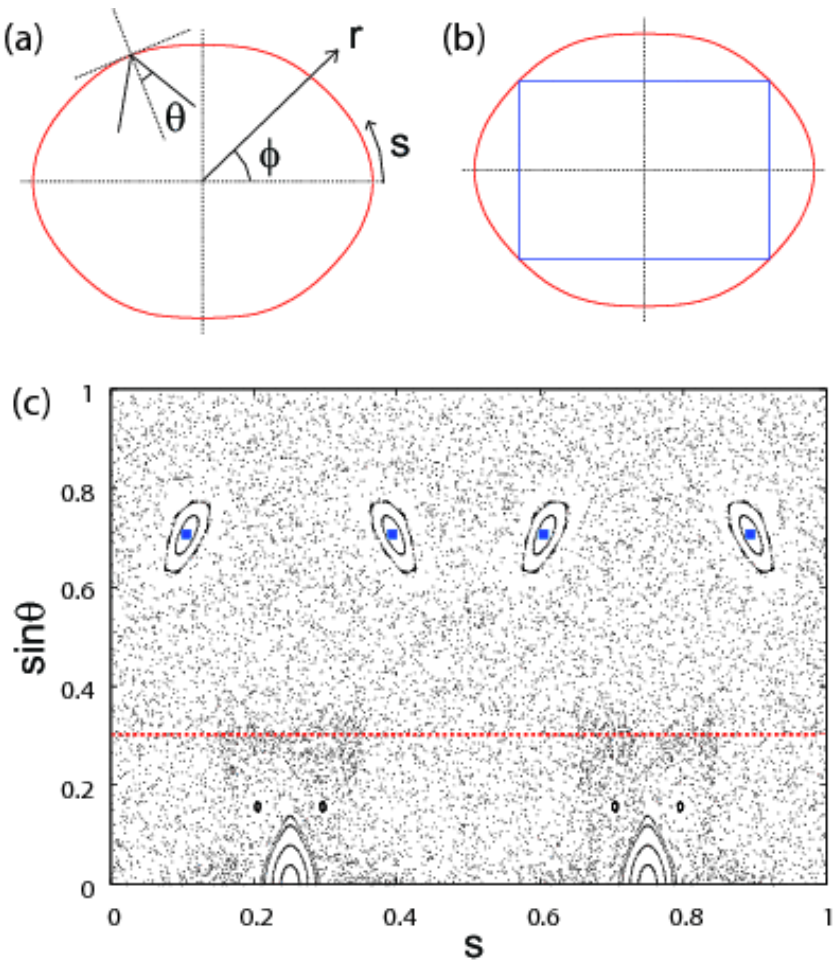

FIG. 1: (color online) (a) The geometry of the asymmetric resonant cavity with the definitions of the polar coordinates $(r, \phi)$ and of the Birkhoff coordinates $(s, \sin \theta)$. (b) The rectangular ray orbit. (c) The upper half of the Poincaré surface of section (PSOS) for the ray dynamics. The coordinate $s$ is normalized by the perimeter of the cavity. The critical line for total internal reflection, $\sin \theta=1 / 3.3$, is plotted by a dotted line. The four filled squares ( $\mathbf{\square})$ represent the rectangular orbit shown in (b).

In this paper, we are interested in quasi-one-dimensional modes strongly localized along the stable rectangular orbit. The existence of such modes can be proven in the short-wavelength limit by the so-called Gaussian optics [29]. This type of modes has been previously studied, for instance, for the so-called 'bow-tie' laser [33], but an important difference here is that the corresponding ray orbit is confined by TIR. Thus, the ray optics based on Fresnel's law as well as the Gaussian optics predict that rectangle-orbit modes are perfectly confined, which is not true for accurate wave description. In order to correctly capture the emission patterns of the rectangleorbit modes, below we resort to numerics.

An accurate way for numerically calculating the modes is provided, for instance, by the boundary element method 34]. In Fig. 2 (a) we present a result obtained by this method, showing the field intensity distribution of a resonant mode strongly localized along the rectangle orbit, where one can see a regular modal pattern along the orbit. Its wavenumber is $k=49.94-i 0.00012$. Concerning the resonant mode calculation, we note that the average radius of the cavity is fixed as $r_{0}=1$. We employ the boundary condition for the transverse-electric 
polarization, to be consistent with experimental data presented later.

In order to observe exponentially small emission from the rectangle-orbit mode, we plot the field intensity distribution in log scale in Fig. 2 (b), where one finds that dominant emission occurs on both sides of the cavity towards the directions $\phi= \pm 90^{\circ}$. These emissions are understood as the wave tunneling effect intrinsic to a raychaotic cavity. This emission mechanism is better explained by the PSOS than the real space. Thus, we consider the PSOS-representation of an eigenfunction called the Husimi distribution [35, 36]. Figure 3 (a) shows the Husimi distribution of the rectangle-orbit mode shown in Fig. 2 (a), where it is plotted in log scale to see exponentially small intensities in the area of the chaotic sea. In Fig. 3 (a), the intensity increases as the color changes from black to white. Reflecting the fact that the mode is localized along the rectangular orbit, we find high intensities on the corresponding islands. In addition, we find some intensities spread over the chaotic sea, even reaching down to the critical lines for TIR, $\sin \theta= \pm 1 / 3.3$. The four high-intensity spots on the critical line labeled $a, b, c$, and $d$ correspond to the four dominant emissions observed in Fig. 2 (b). Together with the field intensity distribution in the real space (Fig. 2 (b)), the Husimi distribution reveals that emission occurs at two close but different boundary points (one towards $\phi=90^{\circ}$ and the other towards $\phi=-90^{\circ}$ ) on each side of the cavity.

The emission pattern of the rectangle-orbit mode can be qualitatively explained in the following manner [26, 27]. Although intensities are highly localized on the islands corresponding to the rectangular orbit, some intensities are leaked to their neighboring chaotic orbits via dynamical tunneling. Once this happens, the leaked intensities are transported to the leaky region (i.e., $|\sin \theta|<1 / 3.3)$ by the chaotic dynamics, resulting in refractive emission. Hence, it is the ray dynamics around the critical lines for TIR that determines the emission pattern. In fact, such ray dynamics is governed by the

(a)

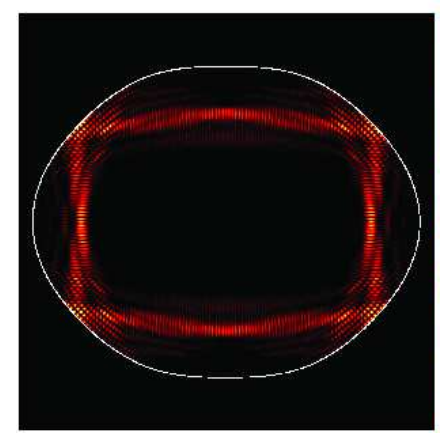

(b)

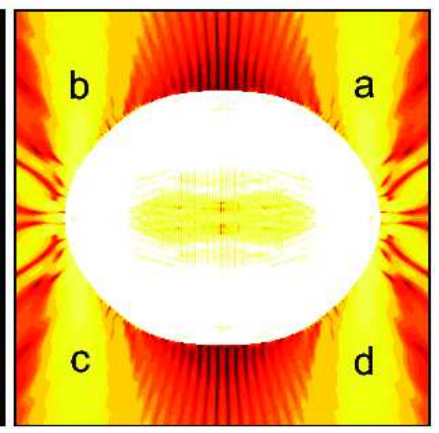

FIG. 2: (color online) (a) Field intensity distribution of the rectangle-orbit mode with the wavenumber $k=49.94-$ $i 0.00012$. (b) Log-scale plot of the rectangle-orbit mode. The intensity increases as the color changes from black to white. unstable manifolds emanating from a short-periodic unstable periodic point located near the critical line 37 41]. In Fig. 3 (b), we show how a ray ensemble initially prepared around the leftmost island is mapped to after 4 bounces. The initially circular area is stretched and folded after 4 bounces, partially penetrating into the region below the critical line as magnified in the inset of Fig. 3 (b). As illustrated in Fig. 3] (a), the high-intensity spot labeled $c$ coincides with the position where the unstable manifold crosses the critical line. The positions of
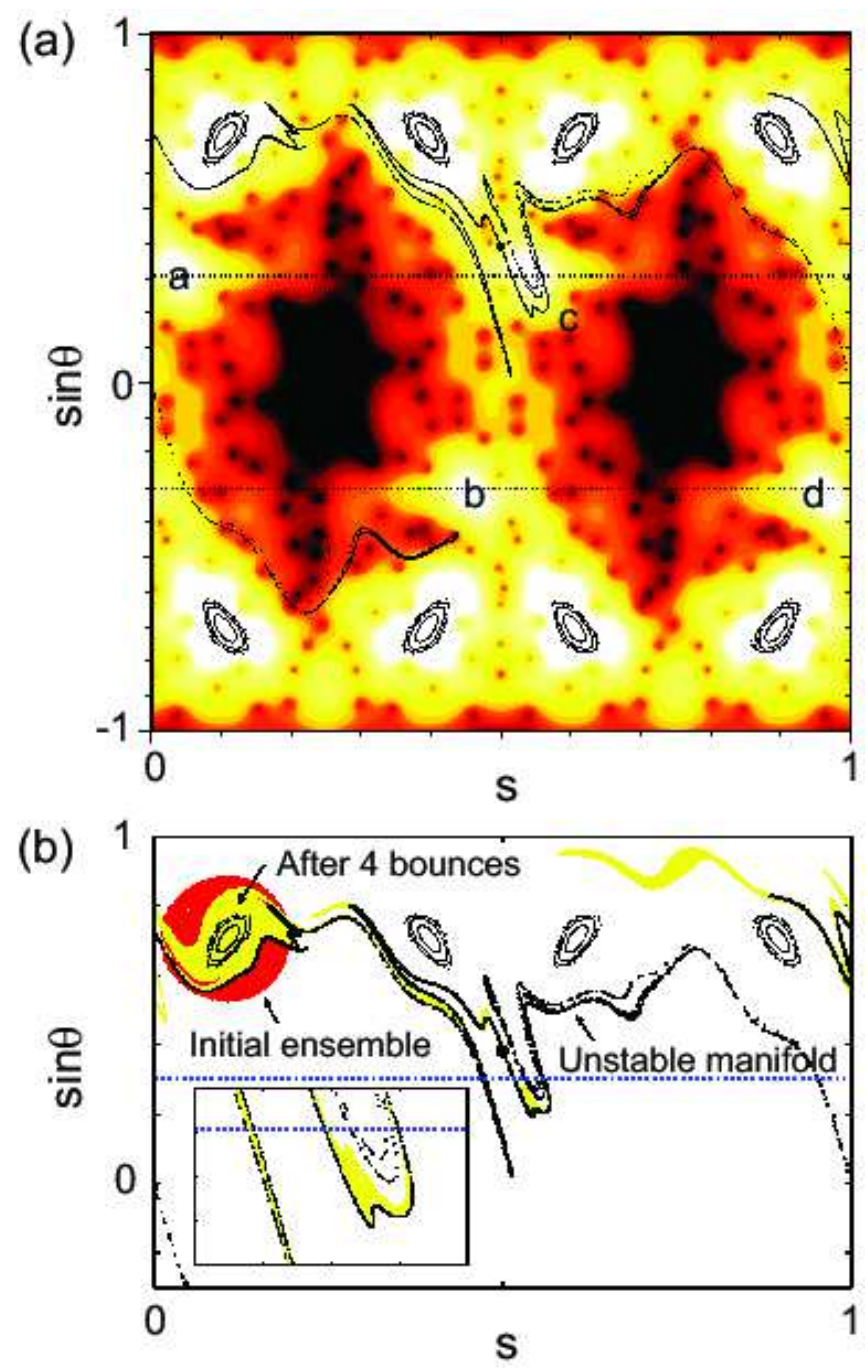

FIG. 3: (color online) (a) Log-scale plot of the Husimi distribution for the rectangle-orbit mode with $k=49.94-i 0.00012$, where the islands corresponding to the rectangular orbit and the unstable manifolds of the unstable period-3 point $(0.5,0.389 \ldots)$ are superimposed. The intensity increases as the color changes from black to white. The critical lines for total internal reflection, $\sin \theta= \pm 1 / 3.3$, are plotted by dotted lines. (b) Ray-dynamical evolution of an ensemble initially prepared over the leftmost island and its neighborhood in the upper half PSOS. The inset shows a magnification around the area where the unstable manifold overlaps with the critical line. 
the other high-intensity spots $(a, b$ and $d)$ are explained in the same manner.

In the remaining part of this paper, we study whether the theoretically predicted chaos-assisted emission from the rectangle-orbit modes can be experimentally observed. Therefore, it is crucial in experiments to excite only the rectangle-orbit modes, suppressing the excitation of the other modes. In order to achieve this selective excitation, we employ the selective pumping method that has been previously examined for quasi-stadium-shaped microcavity lasers, where the selective excitation of two different types of modes, called the axis modes and ring modes, has been successfully demonstrated [42 48].

\section{DEVICE STRUCTURE}

In this section, we explain the device structure to achieve the selective excitation (see Ref. [43] for the details of the fabrication process). Figure 4 shows a schematic cross-sectional view of the device. The device is fabricated from MOCVD-grown material with a $1.5-\mu \mathrm{m} n-\mathrm{Al}_{0.5} \mathrm{Ga}_{0.5} \mathrm{As}$ lower cladding layer, a $0.2-\mu \mathrm{m}$ $n-\mathrm{Al}_{x} \mathrm{Ga}_{1-x} \mathrm{As}(x=0.5-0.2)$ graded region, a $10-\mathrm{nm}$ GaAs quantum well layer, a $0.2-\mu \mathrm{m} p-\mathrm{Al}_{x} \mathrm{Ga}_{1-x} \mathrm{As}(x=$ $0.2-0.5)$ graded region, a $1.5-\mu \mathrm{m} p-\mathrm{Al}_{0.5} \mathrm{Ga}_{0.5} \mathrm{As}$ upper cladding layer, a $0.2-\mu \mathrm{m} p$-GaAs contact layer, and a $400-\mathrm{nm} \mathrm{SiO}_{2}$ layer. The average radius $r_{0}$ of the device is fixed as $50 \mu \mathrm{m}$.

The key structure for the selective excitation is the "contact window" etched to the $\mathrm{SiO}_{2}$ layer. The $\mathrm{SiO}_{2}$ layer works as the insulation between the semiconductor layers and the $p$-electrode metal, enabling a selective current injection through the contact window. The top-view shape of the $p$-GaAs layer is given by adding $1-\mu \mathrm{m}$ margins to the regions of the contact window which is formed by a dry etching process before depositing the $\mathrm{SiO}_{2}$ layer.

In order to excite only the rectangle-orbit modes, we form the contact window along the rectangular orbit as illustrated in Figs. 5 (a)-(c). It is not known a priori what design of the contact window is most optimal for stable and efficient excitation of the rectangle-orbit modes. To

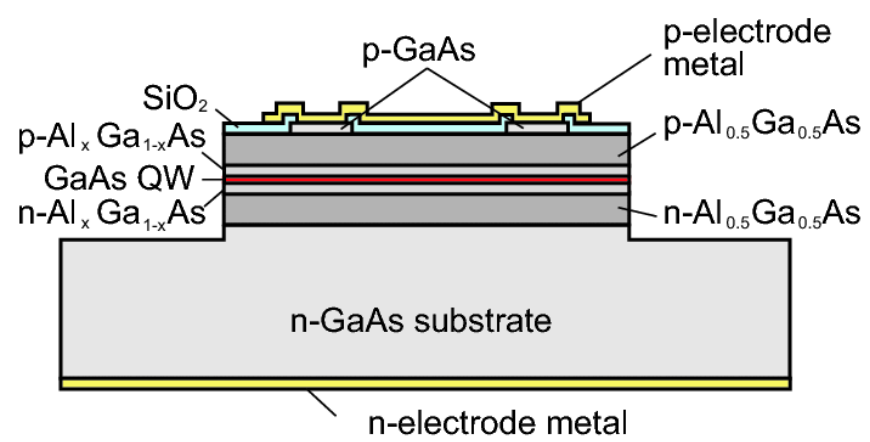

FIG. 4: (color online) Schematic cross-sectional view of the device. test the sensitivity of the selective excitation on a contact window pattern, we examine three different patterns of the contact window, i.e., a broad, an intermediate, and a narrow one, respectively shown in Figs. 5 (a), 5] (b), and 5(c). We show in Fig. 5 (e) how the narrow contact window overlaps with the rectangle-orbit mode.

On the rim of the cavity, we make the "margin area" where $\mathrm{SiO}_{2}$ is left. This is to avoid excitation of whispering-gallery-type modes that are localized along the rim of the cavity. The precise shape of the margin area is given by $0.75 R(\phi) \leq r \leq R(\phi)$. The $p$-electrode metal is deposited over the contact window and part of
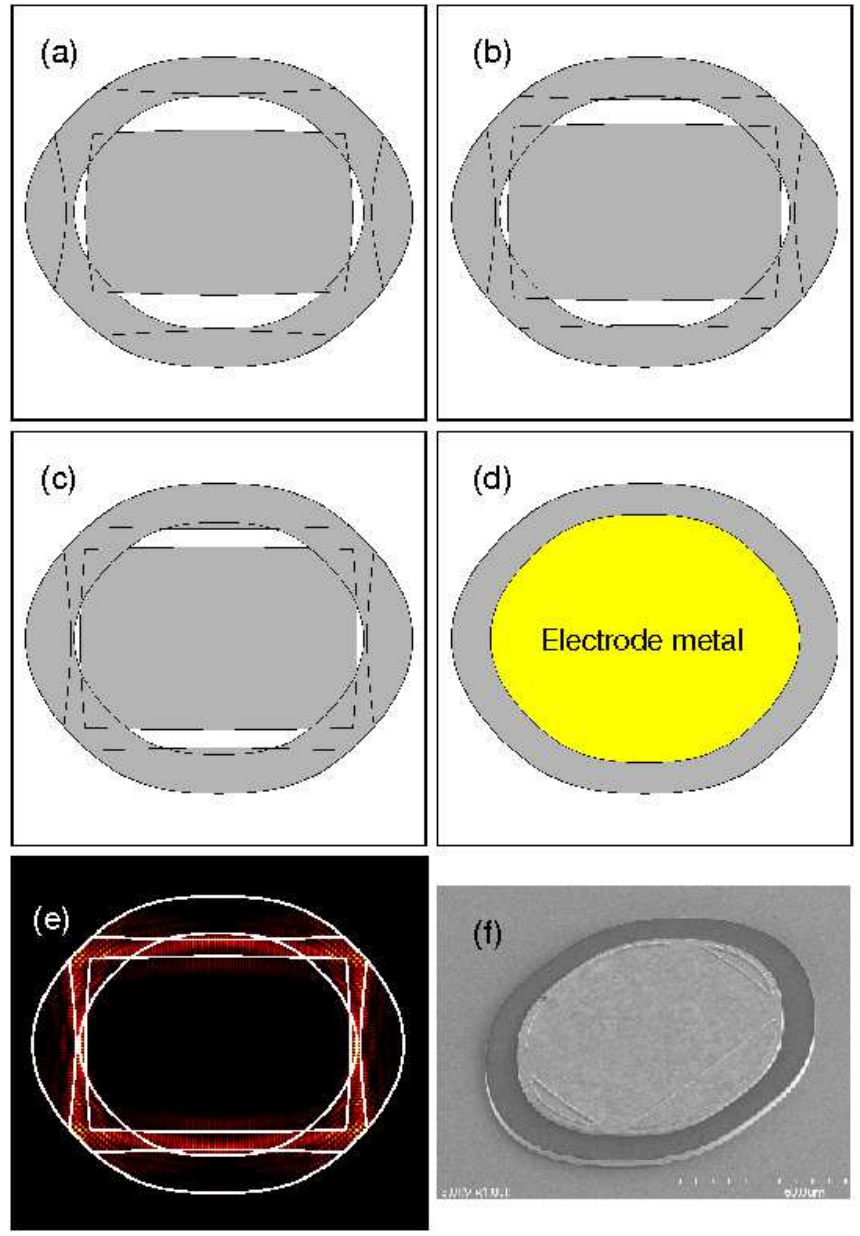

FIG. 5: (color online) (a)-(c): Top-view geometry of the $\mathrm{SiO}_{2}$ layer. The white regions inside the cavity define the contact window where $\mathrm{SiO}_{2}$ is removed and through which currents are injected. The contact window is broad in (a), intermediate in (b), and narrow in (c). We make the "margin area" on the rim, whose shape is defined by $0.75 R(\phi) \leq r \leq R(\phi)$. Above the $\mathrm{SiO}_{2}$ layer, the $p$-electrode metal is deposited as in (d), whose shape is defined by $r \leq 0.8 R(\phi)$. (e) Field intensity distribution of the rectangle-orbit mode with $k=49.94-$ $i 0.00012$ superimposed with the narrow contact window. (f) Scanning electron microscope image of a fabricated device with the broad contact window. The average radius is $r_{0}=50$ $\mu \mathrm{m}$. 
the surrounding $\mathrm{SiO}_{2}$ layer as shown in Fig. 5 (d) and its shape is defined by $r \leq 0.8 R(\phi)$. In Fig. [5(f), we show the scanning electron microscope image of a fabricated device with the broad contact window.

\section{SPECTRAL CHARACTERISTICS}

As, for instance, the Gaussian optic theory directly demonstrates [29], rectangle-orbit modes are regarded as the quantization along the rectangular orbit. Their longitudinal mode spacing is given by

$$
\triangle \lambda=\frac{\lambda^{2}}{n L\left(1-\frac{\lambda}{n} \frac{d n}{d \lambda}\right)},
$$

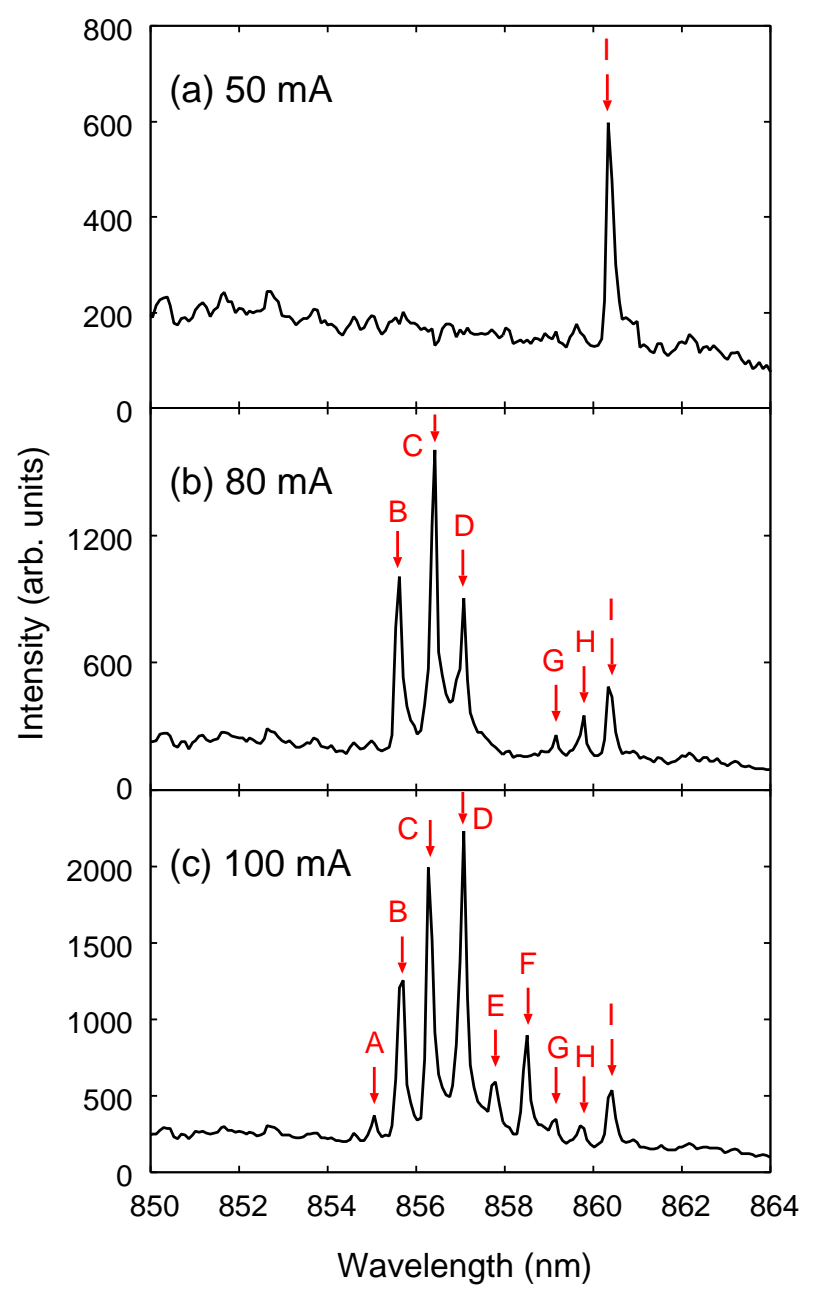

FIG. 6: (color online) Lasing spectra of the device with the narrow contact window for different pumping currents: (a) 50 mA; (b) $80 \mathrm{~mA}$; (c) $100 \mathrm{~mA}$. Equidistantly spaced peaks are observed in (c), where the average mode spacing is $0.67 \mathrm{~nm}$, in good agreement with the longitudinal mode spacing of the rectangle-orbit modes, $0.63 \mathrm{~nm}$. where $\lambda$ is the wavelength, $n$ the effective refractive index, $L$ the path length of the rectangle orbit, and $d n / d \lambda$ the dispersion. In this section, we present measured spectral data showing equidistantly spaced peaks corresponding to the above mode spacing, which constitutes definitive evidence that rectangle-orbit modes only are excited. The devices are tested in pulsed mode operation at $25^{\circ} \mathrm{C}$ with pulse width $500 \mathrm{~ns}$ and repetition rate $1 \mathrm{kHz}$. In Fig. 6, we show lasing spectra of the device with the narrow contact window for the pumping currents $50 \mathrm{~mA}, 80 \mathrm{~mA}$, and $100 \mathrm{~mA}$. For $50 \mathrm{~mA}$, which is just above the lasing threshold current, one can confirm single-mode lasing. For $100 \mathrm{~mA}$, the spectrum shows equidistantly spaced peaks with the averaged mode spacing $\Delta \lambda=0.67 \mathrm{~nm}$. Considering the uncertainty of the refractive index and the resolution of the measurement, one can conclude that this value corresponds to the longitudinal mode spacing of the rectangle-orbit modes estimated as $0.63 \mathrm{~nm}$. This theoretical estimate is calculated from Eq. (2) with $\lambda=860 \mathrm{~nm}, n=3.3, L=283.04 \mu \mathrm{m}$, and $d n / d \lambda=-1.0 \mu \mathrm{m}^{-1}$. Here we use the value of the dispersion $d n / d \lambda$ from Ref. [49].

Because currents are not injected around the rim of the cavity, the lasing of whispering-gallery-type modes is highly unlikely. This can be confirmed by estimating the mode spacing corresponding to whispering-gallery-type modes. Using the above formula with $L=317.75 \mu \mathrm{m}$ (i.e., perimeter of the cavity), one can estimate their mode spacing as $0.56 \mathrm{~nm}$, which is considerably smaller than the measured mode spacing.

The above theoretical estimates convince us that the equidistantly spaced peaks in Fig. 6 (c) are the rectangleorbit modes, not the whispering-gallery-type modes. In

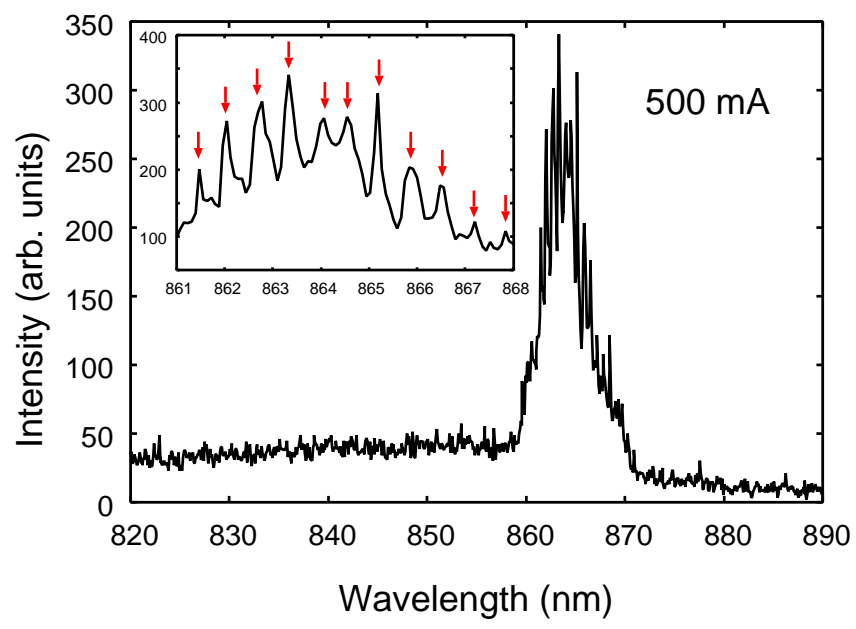

FIG. 7: (color online) Lasing spectrum of the device with the narrow contact window for the pumping current $500 \mathrm{~mA}$. The inset shows the magnification of a high-intensity range, where equidistantly spaced peaks are observed, whose average mode spacing is $0.64 \mathrm{~nm}$, in excellent agreement with the longitudinal mode spacing of the rectangle-orbit modes, 0.63 nm. 
addition, using Fig. 6 (c) as a reference, we can also conclude that peaks observed in Figs. [6 (a) and (b) correspond to the rectangle-orbit modes.

We confirmed selective excitation of the rectangle-orbit modes for a much higher pumping current. In Fig. 77 we show the lasing spectra for the pumping current $500 \mathrm{~mA}$, where one finds again equidistantly spaced peaks with the average mode spacing $0.64 \mathrm{~nm}$, which is in excellent agreement with the theoretical estimate $0.63 \mathrm{~nm}$. The spectral peaks in Fig. 7 are red-shifted from those in Fig. 6, which is considered to be due to a heat effect caused by the high pumping current.

Concerning the lasing of higher transverse excited modes, we cannot find its clear evidence for lower pumpings. However, for the pumping $500 \mathrm{~mA}$, we can identify subpeaks at, for instance, $861.7 \mathrm{~nm}$ and $862.4 \mathrm{~nm}$, which are considered to be higher transverse excitations.

We also confirmed that the selective excitation is achieved with the intermediate and broad contact windows. Figures 8 (a) and 8 (b) show spectra for the device with the intermediate contact window at $140 \mathrm{~mA}$ and for the device with the broad contact window at $80 \mathrm{~mA}$, respectively. In both cases, one can confirm equidistantly spaced peaks. The average mode spacing is $0.67 \mathrm{~nm}$ in

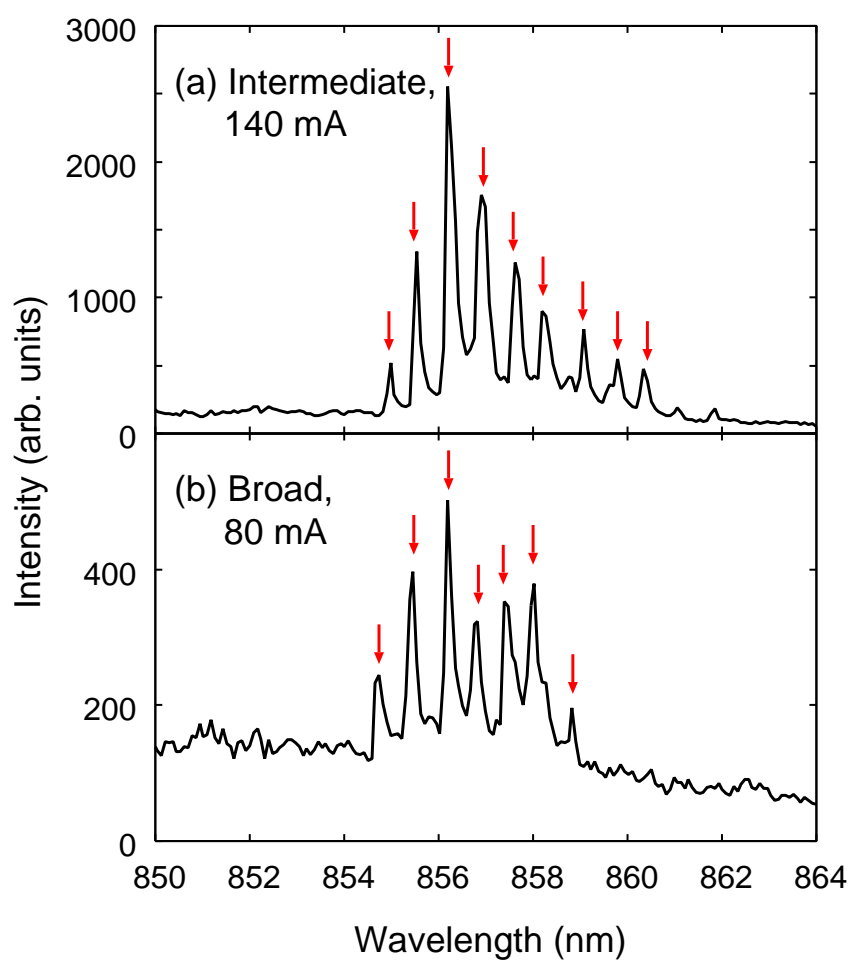

FIG. 8: (color online) Lasing spectra for (a) the device with the intermediate contact window at the pumping current 140 $\mathrm{mA}$ and for (b) the device with the broad contact window at $80 \mathrm{~mA}$. Equidistantly spaced peaks are observed in both cases. The average mode spacing is $0.67 \mathrm{~nm}$ in (a), while $0.68 \mathrm{~nm}$ in (b), both in good agreement with the longitudinal mode spacing of the rectangle-orbit modes, $0.63 \mathrm{~nm}$.
Fig. 8 (a) while $0.68 \mathrm{~nm}$ in Fig. 8 (b). These values are again in good agreement with the theoretical estimate $0.63 \mathrm{~nm}$. As for the lasing threshold currents, we could not find significant dependence on the contact window patterns, i.e., the threshold currents are around $50 \mathrm{~mA}$ irrespective of the contact window pattern.

\section{EMISSION CHARACTERISTICS}

For microcavities with mostly or fully chaotic ray dynamics, it has been reported that experimental far-field patterns are well explained by ray-dynamical calculations 40, 43, 50 53. Generally, some intrinsic deviation is observed between the far-field pattern of a single resonant mode and that of ray-dynamical calculation, especially in the short-wavelength regime [51 54]. Nonetheless, it was demonstrated that the averaged far-field pattern of many resonant modes is very well reproduced by raydynamical calculation. Because multi-mode lasing works as a mechanism to realize the "average" of many modes, one can expect that the correspondence between experimental data and ray-dynamical calculation becomes the better, the more the number of lasing modes increases. In fact, the validity of this expectation was confirmed for a stadium-shaped microlaser [52]. In this section, we report measured emission patterns of our devices where the rectangle-orbit modes are selectively excited and show to which extent they can be reproduced by ray-dynamical calculation.

We show far-field patterns of the device with the narrow contact window for the pumping currents $100 \mathrm{~mA}$ and $500 \mathrm{~mA}$ in Figs. 9 (a) and 9 (b), respectively. We note that these patterns are normalized after subtracting uniform background due to spontaneous emission. In good accordance with the theoretical prediction based on the chaos-assisted emission, we observe strong emissions towards $\pm 90^{\circ}$.

In Figs. 9 (a) and 9 (b), we superpose the theoretical far-field pattern obtained by ray-dynamical calculation. We find that the experimental far-field pattern for 500 $\mathrm{mA}$ is well reproduced by ray-dynamical calculation even for the substructures of the main peaks. As shown in Ref. [30], wave calculation of the far-field pattern for a single mode does not show such substructures (see Fig. 5 of Ref. [30]). Therefore we conclude that the substructures of the main peaks in the measured far-field pattern are due to multimode lasing, because the far-field pattern for ray-dynamical calculation can be considered as that from an average of many modes as explained above.

For $100 \mathrm{~mA}$, the experimental far-field pattern is asymmetric, showing some deviations from the ray-dynamical calculation. An intrinsic mechanism leading to the asymmetry is the locking of modes belonging to different parities [48, [55]. The locking is caused by nonlinear modal interaction due to the lasing medium, creating a lasing mode whose emission pattern violates the symmetries of the cavity. When the number of such a lasing mode 


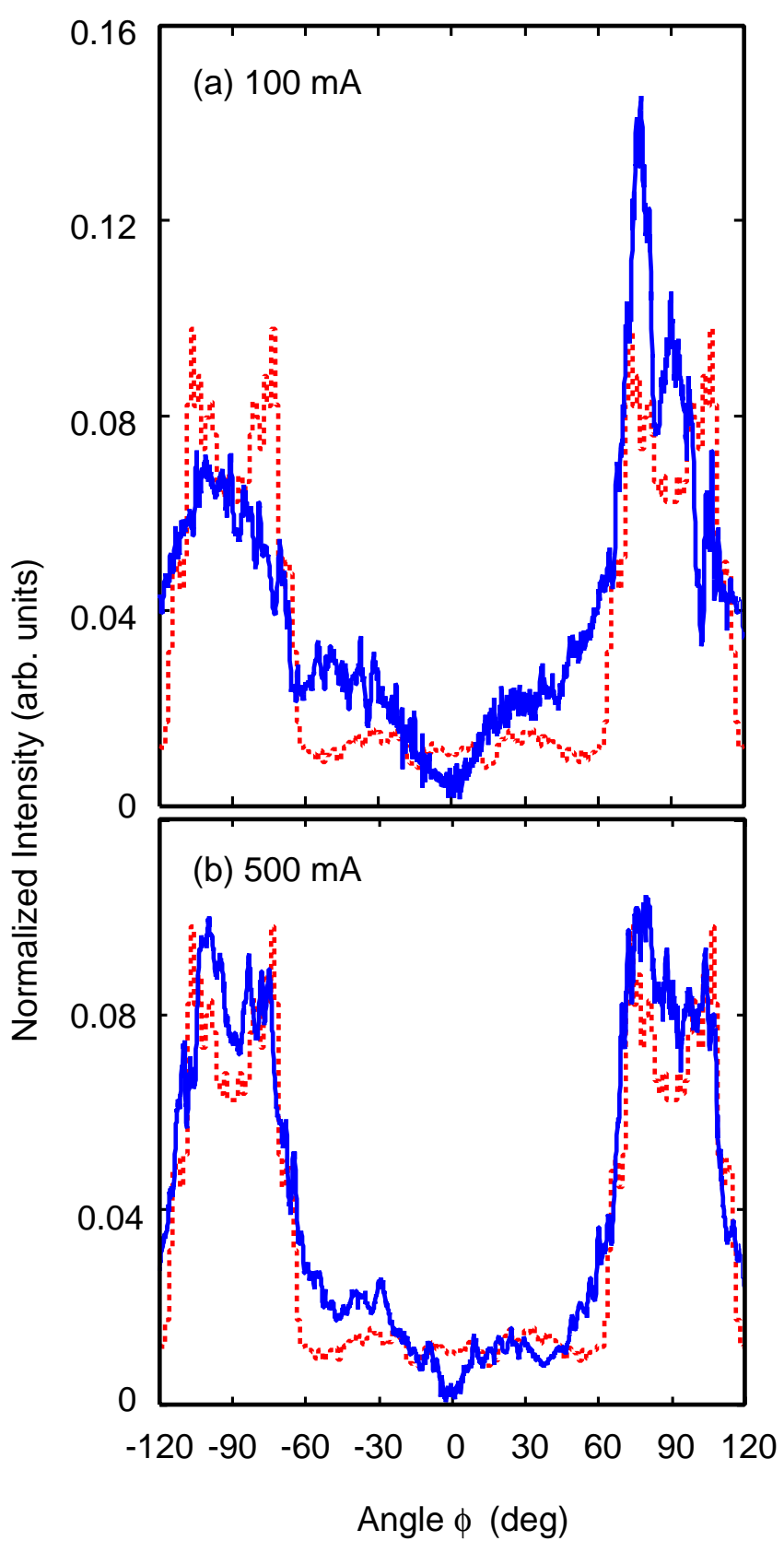

FIG. 9: (color online) Far-field emission patterns of the device with the narrow contact window for (a) $100 \mathrm{~mA}$ and (b) 500 $\mathrm{mA}$. The definition of the angle $\phi$ is given in Fig. 1 (a). The solid curves represent experimental data, while the dotted curves represent ray-dynamical data.

is large, the asymmetry is averaged out, resulting in a symmetric emission. However, the asymmetry becomes prominent when the number of such a mode is relatively small [55]. For the pumping $500 \mathrm{~mA}$, we can identify many modes with significant intensities in the lasing spectrum shown in Fig. 7, while for the pumping $100 \mathrm{~mA}$ we find only a couple of dominant lasing modes in the lasing spectrum shown in Fig. 6 (c). We expect that for $100 \mathrm{~mA}$ the relatively small number of the lasing modes
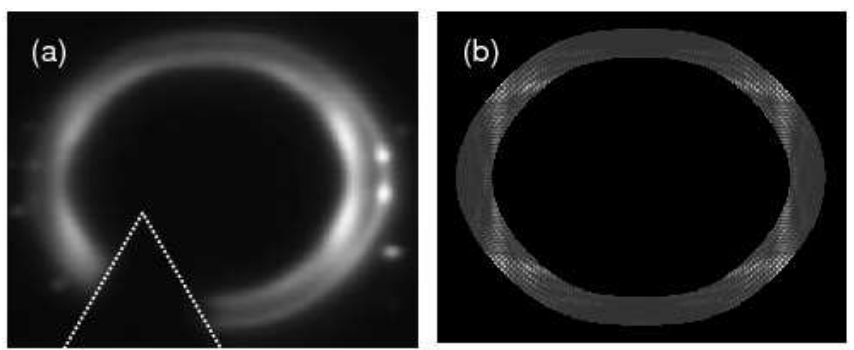

FIG. 10: (a) CCD photo of the lasing device with the broad contact window for $300 \mathrm{~mA}$. The photo is taken from a slightly oblique angle, causing the difference between the right hand edge and left hand edge. The outline of a needle for current injection is indicated by the dotted line. (b) The field intensity distribution of the rectangle-orbit mode shaded by the electrode metal area.

makes the emission asymmetry significant.

Lastly, we show a CCD photo of the lasing device with the broad contact window at $300 \mathrm{~mA}$ in Fig. 10 (a). Although the lasing emission is in-plane, some light scattered inside the cavity is observed from the top in the margin area. For comparison, we show in Fig. 10 (b) the field intensity distribution of the rectangle-orbit mode shaded by the electrode metal area. We can see that bright regions in Fig. 10 (a) are coming mainly from the four corners of the rectangular orbit. Moreover, we can observe two bright spots around the right hand edge of the cavity. These are considered to be the light scattered at the cavity boundary, thus indicating the emission points. These emission points are in good accordance with the theoretical prediction drawn from the Husimi distribution shown in Fig. 3 (a) as well as the near-field pattern shown in Fig. 2 (b).

\section{CONCLUSION}

We have focused on the quasi-one-dimensional modes of the asymmetric resonant cavity, which are strongly localized along the stable rectangular ray orbit that is confined by TIR. Direct numerical calculation of such a resonant mode revealed that directional emission occurs from cavity boundary points apart from the rectangular ray orbit. This at first sight counterintuitive result was explained by the chaos-assisted emission mechanism consisting of regular-to-chaotic tunneling and subsequent chaotic transport leading to refractive emission.

We fabricated the cavity with the structure to selectively excite the rectangle-orbit modes. The success of the selective excitation was confirmed by the observation of the equidistantly spaced peaks in the lasing spectra well corresponding the longitudinal mode spacing of the rectangle-orbit modes. In the emission patterns, we observed directional emission attributed to the chaosassisted emission.

Our results demonstrated that the concepts of quan- 
tum/wave chaos and nonlinear dynamics such as dynamical tunneling and unstable manifolds are indispensable for precisely understanding phenomena occurring in the micro devices. As a way to extract light from optical cavities, the chaos-assisted directional emission adds a new idea to the conventional theories [56], and it would be of interest to apply this idea to design practical devices.

\section{Acknowledgments}

Shinohara and Hentschel acknowledge financial support from the DFG research group 760 "Scattering Systems with Complex Dynamics" and the DFG Emmy Noether Program.
[1] Y. Yamamoto and R. E. Slusher, Phys. Today 46, 66 (1993).

[2] Optical Processes in Microcavities, edited by R. K. Chang and A. J. Campillo (World Scientific, 1996).

[3] K. J. Vahala, Nature (London) 424, 839 (2003).

[4] Optical Microcavities, edited by K. Vahala (World Scientific, 2004).

[5] J. U. Nöckel and A. D. Stone, in Optical Processes in Microcavities, edited by R. K. Chang and A. J. Campillo (World Scientific, 1996).

[6] H. G. L. Schwefel, H. E. Tureci, A. D. Stone, and R. K. Chang, in Optical Microcavities, edited by K. Vahala (World Scientific, Singapore, 2004).

[7] T. Harayama and S. Shinohara, Laser Photonics Rev. 5, 247 (2011).

[8] M. J. Davis and E. J. Heller, J. Chem. Phys. 75, 246 (1981).

[9] S. C. Creagh, in Tunneling in Complex Systems, edited by S. Tomsovic (World Scientific, Singapore, 1998).

[10] J. D. Hanson, E. Ott, and T. M. Antonsen, Phys. Rev. A 29, 819 (1984).

[11] A. Shudo and K. S. Ikeda, Phys. Rev. Lett. 74, 682 (1995).

[12] M. Sheinman, S. Fishman, I. Guarneri, and L. Rebuzzini, Phys. Rev. A 73, 052110 (2006).

[13] A. Bäcker, R. Ketzmerick, S. Löck, and L. Schilling, Phys. Rev. Lett. 100, 104101 (2008).

[14] O. Bohigas, S. Tomsovic, and D. Ullmo, Phys. Rep. 223, 43 (1993).

[15] S. Tomsovic and D. Ullmo, Phys. Rev. E 50, 145 (1994).

[16] E. Doron and S. D. Frischat, Phys. Rev. Lett. 75, 3661 (1995).

[17] F. Leyvraz and D. Ullmo, J. Phys. A: Math. Gen. 29, 2529 (1996).

[18] S. D. Frischat and E. Doron, Phys. Rev. E 57, 1421 (1998).

[19] W. A. Lin and L. E. Ballentine, Phys. Rev. Lett. 65, 2927 (1990).

[20] C. Dembowski, H. -D. Gräf, A. Heine, R. Hofferbert, H. Rehfeld, and A. Richter, Phys. Rev. Lett. 84, 867 (2000).

[21] A. Bäcker, R. Ketzmerick, S. Löck, M. Robnik, G. Vidmar, R. Höhmann, U. Kuhl, and H. -J. Stöckmann, Phys. Rev. Lett. 100, 174103 (2008).

[22] W. K. Hensinger, H. Häffner, A. Browaeys, N. R. Heckenberg, K. Hlmerson, C. McKenzie, G. J. Milburn, W. D. Phillips, S. L. Rolston, H. Rubinsztein-Dunlop, and B. Upcroft, Nature (London) 412, 52 (2001).

[23] D. A. Steck, W. H. Oskay, and M. Raizen, Science 293, 274 (2001).

[24] J. U. Nöckel and A. D. Stone, Nature (London) 385, 45 (1997).

[25] G. Hackenbroich and J. U. Nöckel, Europhys. Lett. 39,
371 (1997).

[26] V. A. Podolskiy and E. E. Narimanov, Opt. Lett. 30, 474 (2005).

[27] E. E. Narimanov and V. A. Podolskiy, IEEE J. Sel. Top. Quantum Electron. 12, 40 (2006).

[28] A. Bäcker, R. Ketzmerick, S. Löck, J. Wiersig, and M. Hentschel, Phys. Rev. A 79, 063804 (2009).

[29] H. E. Tureci, H. G. L. Schwefel, A. D. Stone, and E. E. Narimanov, Opt. Express 10, 752 (2002).

[30] S. Shinohara, T. Harayama, T. Fukushima, M. Hentschel, T. Sasaki, and E. E. Narimanov, Phys. Rev. Lett. 104, 163902 (2010).

[31] A. D. Stone, Nature (London) 465, 696 (2010).

[32] J. Yang, S.-B. Lee, S. Moon, S.-Y Lee, S. W. Kim, T. T. A. Dao, J.-H. Lee, and K. An, Phys. Rev. Lett. 104, 243601 (2010); K. An and J. Yang, arXiv:0912.0164.

[33] C. Gmachl, F. Capasso, E. E. Narimanov, J. U. Nöckel, A. D. Stone, J. Faist, D. L. Sivco, and A. Y. Cho, Science 280, 1556 (1998).

[34] J. Wiersig, J. Opt. A: Pure Appl. Opt. 5, 53 (2003).

[35] B. Crespi, G. Perez, and S. -J. Chang, Phys. Rev. E 47, 986 (1993).

[36] M. Hentschel, H. Schomerus, and R. Schubert, Europhys. Lett. 62, 636 (2003).

[37] H. G. L. Schwefel, N. B. Rex, H. E. Tureci, R. K. Chang, A. D. Stone, T. Ben-Messaoud, and J. Zyss, J. Opt. Soc. Am. B 21, 923 (2004).

[38] S. -Y. Lee, J. -W. Ryu, T. -Y. Kwon, S. Rim, and C. -M. Kim, Phys. Rev. A 72, 061801(R) (2005).

[39] S. Shinohara, T. Harayama, H. E. Türeci, and A. D. Stone, Phys. Rev. A 74, 033820 (2006).

[40] S. -B. Lee, J. Yang, S. Moon, J. -H. Lee, K. An, J. -B. Shim, H. -W. Lee, and S. W. Kim, Phys. Rev. A 75, 011802(R) (2007).

[41] J. Wiersig and M. Hentschel, Phys. Rev. Lett. 100, 033901 (2008).

[42] T. Fukushima, T. Harayama, T. Miyasaka, and P. O. Vaccaro, J. Opt. Soc. Am. B 21, 935 (2004).

[43] T. Fukushima and T. Harayama, IEEE J. Sel. Top. Quantum Electron. 10, 1039 (2004).

[44] T. Fukushima, T. Tanaka, and T. Harayama, Appl. Phys. Lett. 87, 191103 (2005).

[45] M. Choi, T. Tanaka, T. Fukushima, and T. Harayama, Appl. Phys. Lett. 88, 211110 (2006).

[46] T. Fukushima, S. Shinohara, T. Harayama, Opt. Express 15, 17392 (2007).

[47] M. Choi, T. Fukushima, and T. Harayama, Phys. Rev. A 77, 063814 (2008).

[48] T. Harayama, T. Fukushima, S. Sunada, and K. S. Ikeda, Phys. Rev. Lett. 91, 073903 (2003).

[49] H. C. Casey Jr. and M. B. Panish, Heterostructure Lasers (Academic Press, New York, 1978). 
[50] T. Tanaka, M. Hentschel, T. Fukushima, and T. Harayama, Phys. Rev. Lett. 98, 033902 (2007).

[51] S. Shinohara, T. Fukushima, and T. Harayama, Phys. Rev. A 77, 033807 (2008).

[52] M. Choi, S. Shinohara, and T. Harayama, Opt. Express 16, 17554 (2008).

[53] S. Shinohara, M. Hentschel, J. Wiersig, T. Sasaki, and T. Harayama, Phys. Rev. A 80, 031801(R) (2009).
[54] S. Shinohara and T. Harayama, Phys. Rev. E 75, 036216 (2007).

[55] S. Sunada, T. Harayama, and K. S. Ikeda, Phys. Rev. E 71, 046209 (2005).

[56] A. E. Siegman, Lasers (University Science Books, Mill Valley, California, 1986). 